\title{
Evaluation of a high framerate multi-exposure laser speckle contrast imaging setup
}

Martin Hultman, Ingemar Fredriksson, Tomas Strömberg and Marcus Larsson

The self-archived postprint version of this journal publication is available at Linköping University Institutional Repository (DiVA):

http:// urn.kb.se/ resolve?urn=urn:nbn:se:liu:diva- 148844

N.B.: When citing this work, cite the original publication.

Hultman, M., Fredriksson, I., Strömberg, T., Larsson, M., (2018), Evaluation of a high framerate multi-exposure laser speckle contrast imaging setup, High-Speed Biomedical Imaging and Spectroscopy III. https:// doi.org/ 10.1117/ 12.2286248

Original publication available at:

https:/ / doi.org/ 10.1117/ 12.2286248

Copyright: SPIE - International Society for Optical Engineering 


\title{
Evaluation of a high framerate multi-exposure laser speckle contrast imaging setup
}

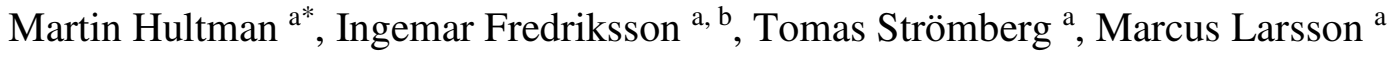 \\ a) Department of Biomedical Engineering, Linköping University, Linköping, Sweden
}

b) Perimed AB, Järfälla-Stockholm, Sweden

\begin{abstract}
We present a first evaluation of a new multi-exposure laser speckle contrast imaging (MELSCI) system for assessing spatial variations in the microcirculatory perfusion. The MELSCI system is based on a 1000 frames per second 1-megapixel camera connected to a field programmable gate arrays (FPGA) capable of producing MELSCI data in realtime. The imaging system is evaluated against a single point laser Doppler flowmetry (LDF) system during occlusionrelease provocations of the arm in five subjects.
\end{abstract}

Perfusion is calculated from MELSCI data using current state-of-the-art inverse models. The analysis displayed a good agreement between measured and modeled data, with an average error below $6 \%$. This strongly indicates that the applied model is capable of accurately describing the MELSCI data and that the acquired data is of high quality. Comparing readings from the occlusion-release provocation showed that the MELSCI perfusion was significantly correlated $(\mathrm{R}=0.83)$ to the single point LDF perfusion, clearly outperforming perfusion estimations based on a single exposure time.

We conclude that the MELSCI system provides blood flow images of enhanced quality, taking us one step closer to a system that accurately can monitor dynamic changes in skin perfusion over a large area in real-time.

Keywords: MELSCI, LSCI, Microcirculation, Blood perfusion

\section{INTRODUCTION}

The microcirculation, i.e. the blood flow in the capillaries, is where the blood exchanges oxygen and nutrients with the cells, and is therefore critical for a healthy body. Impaired microcirculation can be caused by a number of well-known deceases. Consequently, there is a need for fast, accurate devices that can image the microcirculation and follow dynamic changes in real-time. Until recently, such a device has not been possible, simply due to limitations in the available computational devices. With the rise of affordable highly parallel hardware like field programmable gate arrays (FPGA) new possibilities have emerged.

When laser light is backscattered from a static turbid medium, small differences in photon path lengths will give rise to constructive and destructive interference of the light. If the backscattered light is imaged with a camera the result will be a random fringe pattern containing both light and dark spots due to the interference. This speckle pattern will not change in time if the scattering medium is static. However, if the scattering medium is non-static, such as when blood flows through the capillaries in the skin, the speckles will move. These speckle fluctuations originate from Doppler frequency shifts that occur when light is scattered by moving red blood cells (RBCs). The fraction of moving speckles depends directly on the fraction of Doppler shifted light, a property that is governed by the amount of moving $\mathrm{RBCs}^{1}$. Similarly, there is a strong dependency between the speed of the speckle fluctuations and the speed of the RBCs.

Laser speckle contrast imaging (LSCI) quantifies these fluctuations by analyzing the local blurring that occurs when the speckle moves. The degree of blurring depends not only on the amount and magnitude of the Doppler shifted light, but also on the exposure time of the camera ${ }^{2}$. In LSCI the local contrast is used as an inverse measure of speckle blurring. With increasing exposure time, blurring will increase and contrast decrease. Additionally, the decay in contrast with

* Corresponding author: Martin Hultman, Department of Biomedical Engineering, Linköping University, 58183 Linköping, Sweden. martin.o.hultman@liu.se 
exposure time contains information about both RBC flow speed and amount of moving $\mathrm{RBCs}^{3,4}$. It has been suggested that additional information regarding the speed distribution of RBCs can be found in this decay ${ }^{4,5}$.

Current commercial LSCI instruments make use of a fixed exposure time to quantify spatial and temporal variations in tissue perfusion. This evidently limits the possibility for a more comprehensive microcirculatory assessment of the tissue. To overcome this limitation, setups using multi-exposure laser speckle contrasts imaging (MELSCI) have been proposed $^{6,7}$. Typically, these setups have an image acquisition strategy where different exposure times are realized by either changing the camera exposure settings or by using a variable laser pulse width. The acquisition time for these setups is strongly dependent on the number of exposure times being used. Consequently, it is not a viable solution for a real time application operating at high frame rates.

To overcome this, novel approaches based on a high-speed camera connected to a dedicated FPGA processing unit have been proposed ${ }^{8,9}$. We have previously demonstrated an implementation of such a system, where real-time MELSCI data is achieved by cumulative summation of images from a 1000 frames-per-second camera using a fix exposure time of $1 \mathrm{~ms}^{8}$ Initial measurements captured during an occlusion-release provocation of a finger indicated that this system can be used for a fast and accurate acquisition of MELSCI data.

The aim of this study was to assess the microcirculatory response in healthy individuals undergoing occlusion-release provocations using an FPGA based MELSCI technique. MELSCI contrasts were calculated for seven exposure times $1,2,4, . ., 64 \mathrm{~ms}$. Two perfusion measures were calculated based on either a single exposure time or by fitting all exposure times to a three-parameter model for speckle contrast decay, respectively. The perfusion measures were compared to a single-point system for microcirculatory perfusion measurements.

\section{METHOD}

\subsection{Multi-exposure laser speckle contrast imaging}

In LSCI the blurring of the speckle pattern is analyzed over an image by evaluating the contrast in a local area, usually in the range $3 \times 3$ to $7 \times 7$ pixels ${ }^{10}$. In MELSCI this is done for images with different exposure times. In this study, the multiexposure laser speckle contrast was calculated over pixel blocks of size $4 \times 4$ as

$$
K^{2}(T)=\frac{\sigma^{2}(T)}{\langle I(T)\rangle^{2}}
$$

where $T$ denotes the exposure time of the image, and $\sigma$ and $\langle I\rangle$ are the standard deviation and the mean pixel intensity in the $4 \times 4$ block, respectively. In total, 7 exposure times were used; 1, 2, 4, 8, 16, 32, and 64 ms. These were created using a high-speed CoaXPress camera (EoSens 3CXP, Mikrotron, Unterschleißheim, Germany) in conjunction with a field programmable gate array (FPGA) (Kintex7, Xilinx, San Jose, USA). By capturing 64 images with an exposure time of $1 \mathrm{~ms}$ and a negligible inter-frame delay, the FPGA accumulated consecutive images to get longer exposure times from the set of single-exposure images. This resulted in multiple images for all exposure times except the longest (64 ms). Intermediate contrast images were calculated by the FPGA for all these images. The output contrast at $1 \mathrm{~ms}$ was finally calculated as an average of the intermediate 64 contrast values, the output contrast at 2 ms over 32 contrast values and so on. Thus, all the captured data from the 64 images were used for each of the 7 exposure times, which reduces the noise in the images. The huge amount of data - 1-megapixel images at 1000 frames per second (fps) - required the use of special hardware for real-time capture and processing, which was achieved by connecting the camera directly to the FPGA. The MELSCI system and algorithm is presented in detail before ${ }^{8}$.

\subsection{Model and fitting}

Kazmi et.al. ${ }^{11}$ presented a three-parameter model for the decay of speckle contrast with increasing exposure time;

$$
K^{2}(T)=\beta \frac{\rho^{2} \tau^{2}}{2 T^{2}}\left[e^{\frac{-2 T}{\tau}}-1+\frac{2 T}{\tau}\right]+\beta \frac{4 \rho(1-\rho) \tau^{2}}{T^{2}}\left[e^{\frac{-T}{\tau}}-1+\frac{T}{\tau}\right]+\beta(1-\rho)^{2}+v_{\mathrm{n}}
$$

where $\tau$ is the speckle decorrelation time, $\rho$ is the fraction of dynamically scattered light, $\beta$ is a correction factor accounting for the number of speckles in each submatrix, and $T$ is the exposure time in ms. The exposure independent noise offset $v_{\mathrm{n}}$ is assumed to be zero due to noise reduction performed on the contrast data ${ }^{8}$. 
The model was fitted to each multi-exposure pixel in the MELSCI images, using a trust-region-reflective nonlinear optimization (Matlab, MathWorks, Natick, USA) minimizing the mean square error between the model and measured data, with $\tau, \rho$, and $\beta$ as fitting parameters. Part of the processing in the MELSCI system was to calibrate the contrast with a measurement of a stationary paper, for a maximum contrast of 1 . In an ideal setting this would fill the same purpose as $\beta$ in Eq. (2), and we should therefore be able to assume $\beta=1$ for the analysis. However, we observed a model error several orders of magnitude larger when doing so, and thus decided to leave $\beta$ as a free parameter. We will discuss possible reasons for this later in the paper. To reduce the influence of contrast-increasing artifacts such as hair strands a spatial Gaussian filter with $\sigma=1$ and size $5 \times 5$ pixels was applied to the contrast images before the model fitting.

In addition to fitting the multi-exposure contrasts from each sub-matrix, the model was also fitted to the average multiexposure contrast in a $10 \times 10$ region of interest (ROI) in the acquired images. We observed no significant difference between fitting the model to the average contrast in the ROI and fitting the model to each pixel, then averaging the parameters. However, the former is much faster and was thus used for further processing. Before fitting the model to the average contrast, a spike detection algorithm was used to remove movement artifacts. The spikes were detected by finding large increases in the ratio $K^{2}(1) / K^{2}(2)$, followed by an immediate decrease of the same ratio. This indicates a rapid increase in perfusion, followed by a subsequent decrease, which we assume to be due to movement. The actual increase in perfusion during the reperfusion stage of the experiment is, however, not followed by an immediate decrease and was thus ignored by the spike detection. Any spikes found were replaced by linearly interpolating from neighboring values, in order to keep the sample rate of the original data. The threshold for detecting the spikes was selected empirically as a $10 \%$ increase in the ratio.

The MELSCI perfusion estimate was calculated from the model parameters for both the images and graphs as

$$
P=\frac{\rho}{\tau}
$$

The rationale of Eq. (3) is that perfusion is defined as the amount of moving red blood cells times their average speed. The decorrelation time $\tau$ is inversely dependent on the speed, and the fraction of dynamically scattered light $\rho$ is dependent on the amount of moving red blood cells.

In addition to this, the conventional LSCI perfusion estimate ${ }^{12,13}$ was also calculated for comparison as

$$
P_{c}=\frac{1}{K(4)}-1
$$

where $K(4)$ is the single-exposure contrast at $4 \mathrm{~ms}$. The MELSCI processing pipeline, from raw images to perfusion images and graphs, is summarized in Figure 1.

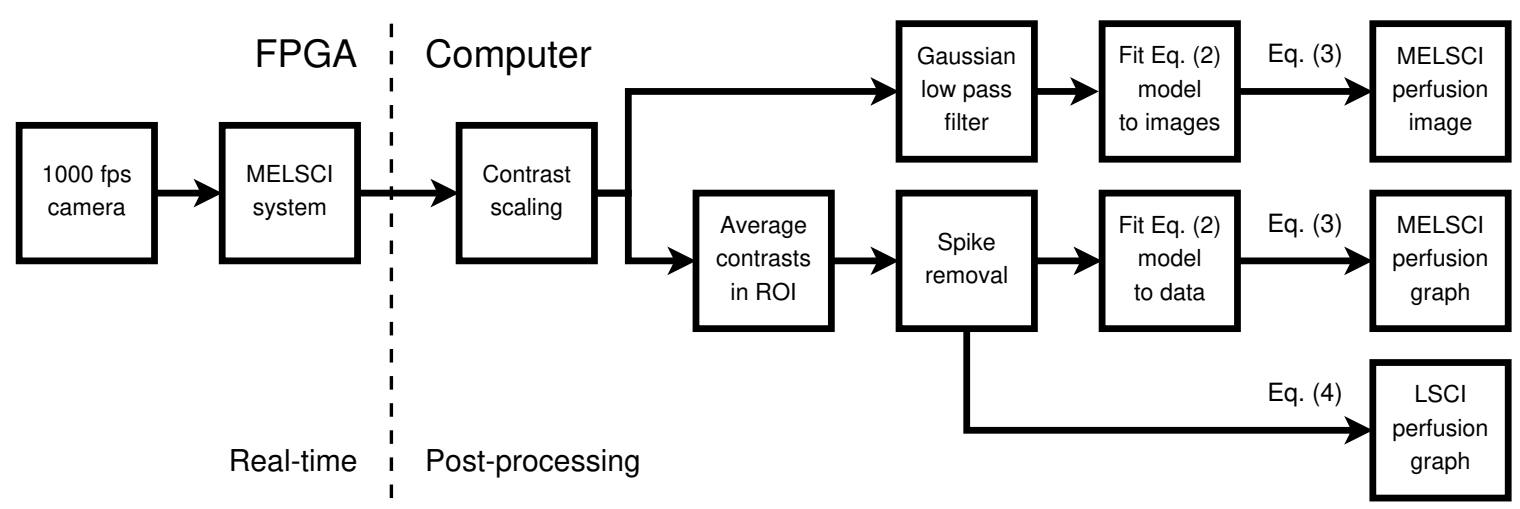

Figure 1: Flow chart of the processing pipeline, from camera to calculated perfusion images and graphs. The left side of the dotted line is the real-time FPGA system and camera described in our previous publication ${ }^{8}$, and the right side is performed in the computer as post processing. 


\subsection{Reference measurements}

A Periflux 6000 EPOS system (enhanced perfusion and oxygen saturation; Perimed AB, Stockholm-Järfälla, Sweden) was used for pointwise reference perfusion measurements. The EPOS system is a fiber-optic probe based instrument that combines laser Doppler flowmetry (LDF) with diffuse reflectance spectroscopy (DRS) for assessment of red blood cell (RBC) tissue fraction, RBC oxygen saturation and speed resolved perfusion. It utilizes a model-based analysis via a joint tissue model and enables quantitative measures. The method is thoroughly described elsewhere. ${ }^{14,15}$ The total quantitative perfusion [\% $\mathrm{RBC} \times \mathrm{mm} / \mathrm{s}$ ] was used as reference to the estimated perfusion value from the MELSCI instrument described above.

\subsection{Experiment setup and protocol}

Five healthy individuals with an age between 22 and 32 years, were subjected to standardized arterial occlusion-release provocations of the forearm at $250 \mathrm{mmHg}$. The protocol was the following; the subject was sitting in a resting position for at least 10 minutes before the actual measurements. Data were recorded for 5 minutes at baseline, 5 minutes of occlusion, and finally 5 minutes of reperfusion. In addition, data from an occlusion-release provocation of a finger was analyzed. That data was collected during a previous study ${ }^{8}$.

During the experiment, the forearm was imaged using the MELSCI system with the camera placed $28 \mathrm{~cm}$ from the arm. Time traces were calculated over a $10 \times 10$ pixel region of interest (ROI) from an area where no visible veins were located. The EPOS probe, was placed on the same arm just outside the camera view.

The measurement protocol was approved by the regional ethical review board at Linköping University, Linköping, Sweden (D.nr 2015/392-31).

\section{RESULTS}

The model was first evaluated on data from our previous publication ${ }^{8}$ in order to qualitatively view the perfusion estimate. The result is shown in Figure 2, where we can observe an obvious change in perfusion in the provoked finger during both occlusion and reperfusion.
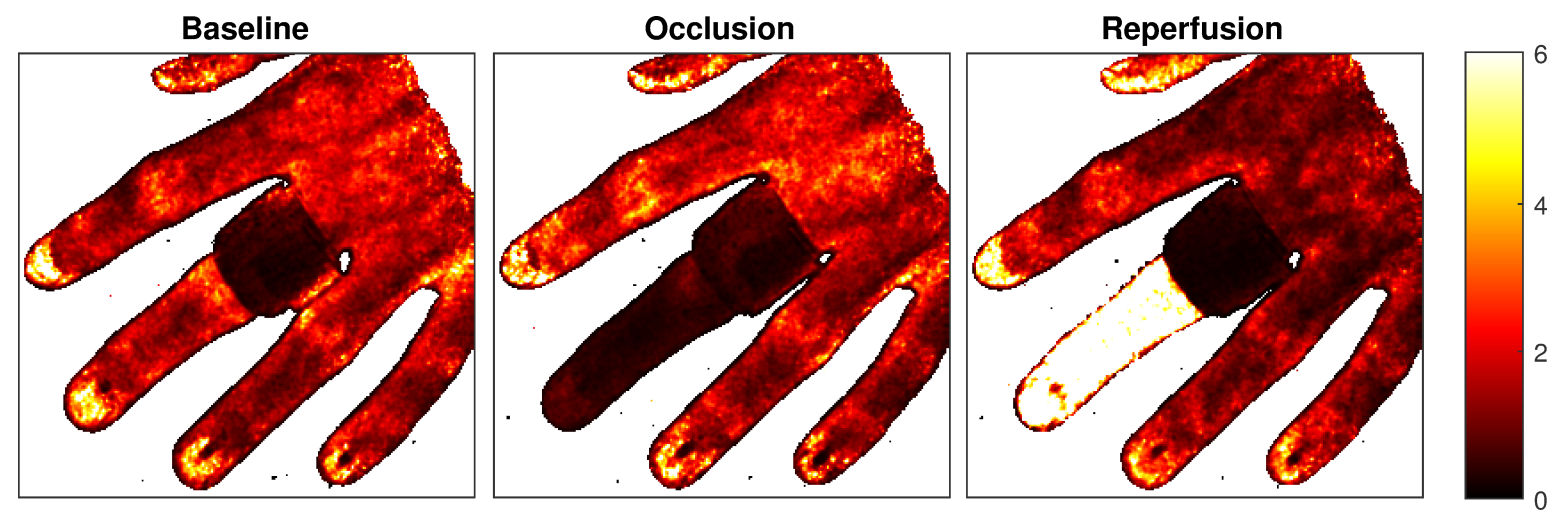

Figure 2: Perfusion (Eq. (3)) of a hand during an occlusion-release provocation. The images were normalized to the perfusion in a $10 \times 10 \mathrm{ROI}$ on the provoked finger during baseline.

Among the 5 subjects in this study, in one case the EPOS probe was not placed correctly. Data from that subject was, therefore, excluded. Figure 3 shows the MELSCI perfusion images during (A) baseline, (B) occlusion, and (C) reperfusion, for one of the subjects. The images were normalized to the average perfusion in a $10 \times 10$ ROI during the first 5 minutes of the experiment. 
(A) Baseline

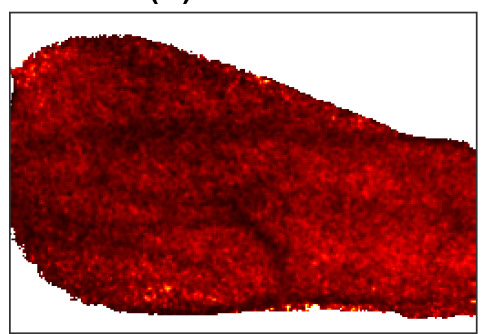

(B) Occlusion

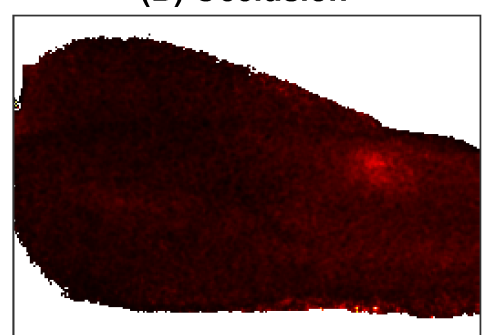

(C) Reperfusion

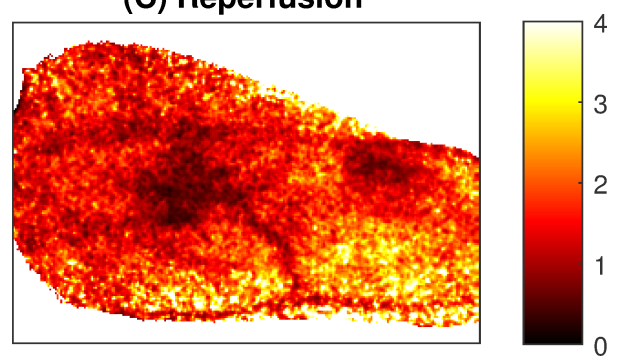

Figure 3: Perfusion (Eq. (3)) images of the forearm during (A) baseline, (B) occlusion, and (C) reperfusion. The images have been normalized to the average baseline perfusion in a $10 \times 10 \mathrm{ROI}$ in a vein-free area.

Figure 4A shows the comparison between MELSCI and EPOS, in a $10 \times 10$ ROI, for one subjects. To be able to accurately compare the MELSCI and EPOS perfusion estimates they were both normalized to their respective baseline (average of $t=20$ to $t=300 \mathrm{~s}$ ). Figure 4B shows the baseline perfusion at $t=145$ to $t=210 \mathrm{~s}$. There is a clear correlation between the MELSCI and EPOS perfusions. The individual parameters extracted from the model in Eq. (2) can be seen in Figure 4C.

(A) MELSCI vs. EPOS perfusion

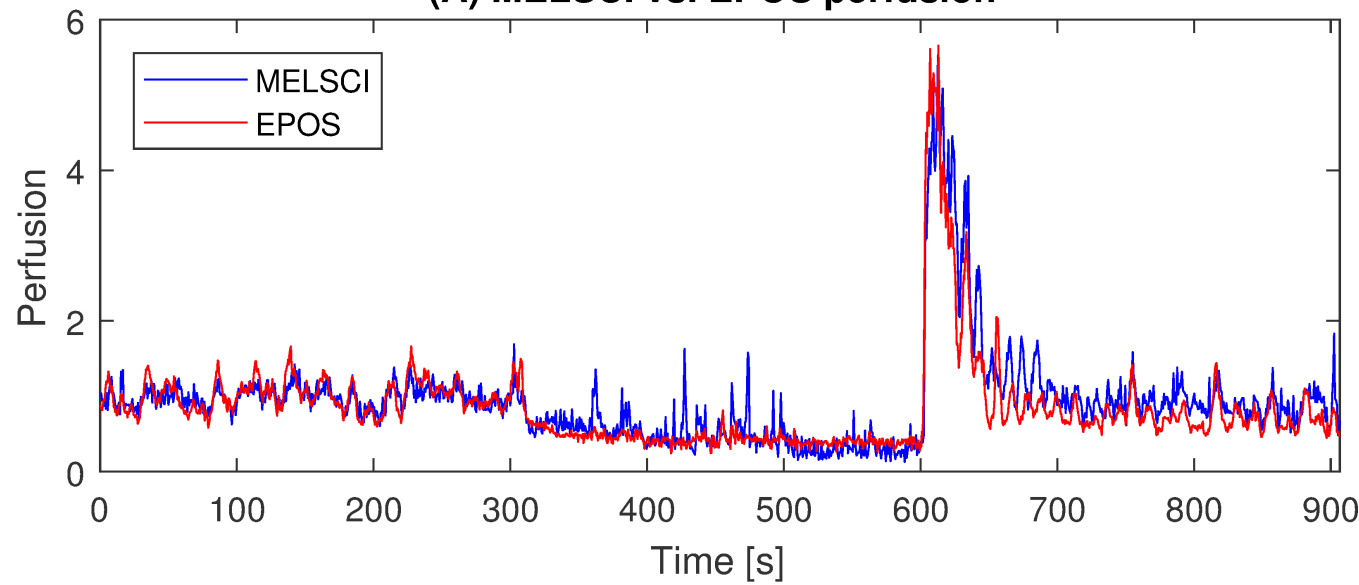

(B) Zoom of (A)

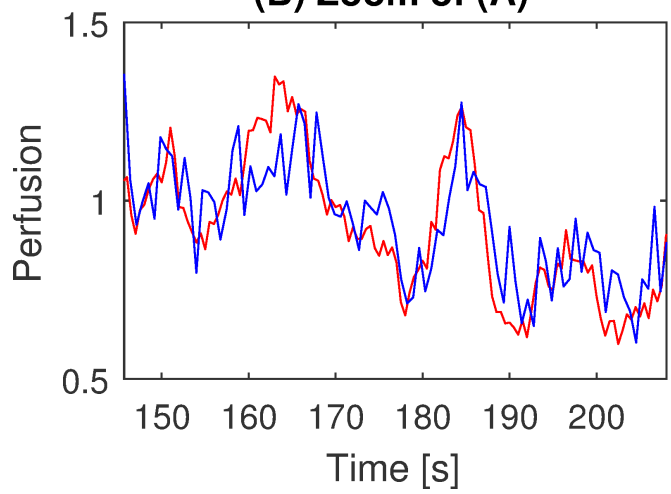

(C) Fitted model parmeters

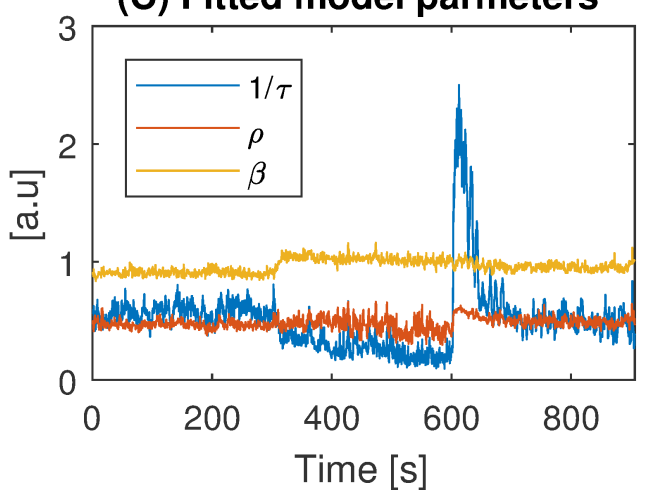

Figure 4: (A) Perfusion graphs for MELSCI and EPOS systems, normalized to their respective baselines. Some movement artifacts remain in the data, as can be seen during the occlusion ( $t=300$ to $t=600 \mathrm{~s})$. (B) Baseline perfusion for $\mathrm{t}=145$ to $\mathrm{t}=210 \mathrm{~s}$, to further emphasize the correlation between the two perfusion estimates. (C) The individual parameters that are extracted from the model in Eq.(2), and combined as in Eq. (3) to give the MELSCI perfusion in (A).

Figure 5 shows three examples of the model fit (A), and (B) the average relative error of the model for each exposure time, calculated as $K_{\text {model }}^{2} / K_{\text {measure }}^{2}-1$, with equal number of samples taken from all subjects (total N=26104). From 
each of the subjects 5 ROIs were selected on the arm, making sure they sampled as different areas as possible, while still avoiding veins. The error bars indicate one standard deviation from the average. On average the model error was $<6 \%$ for all subjects and all time points.
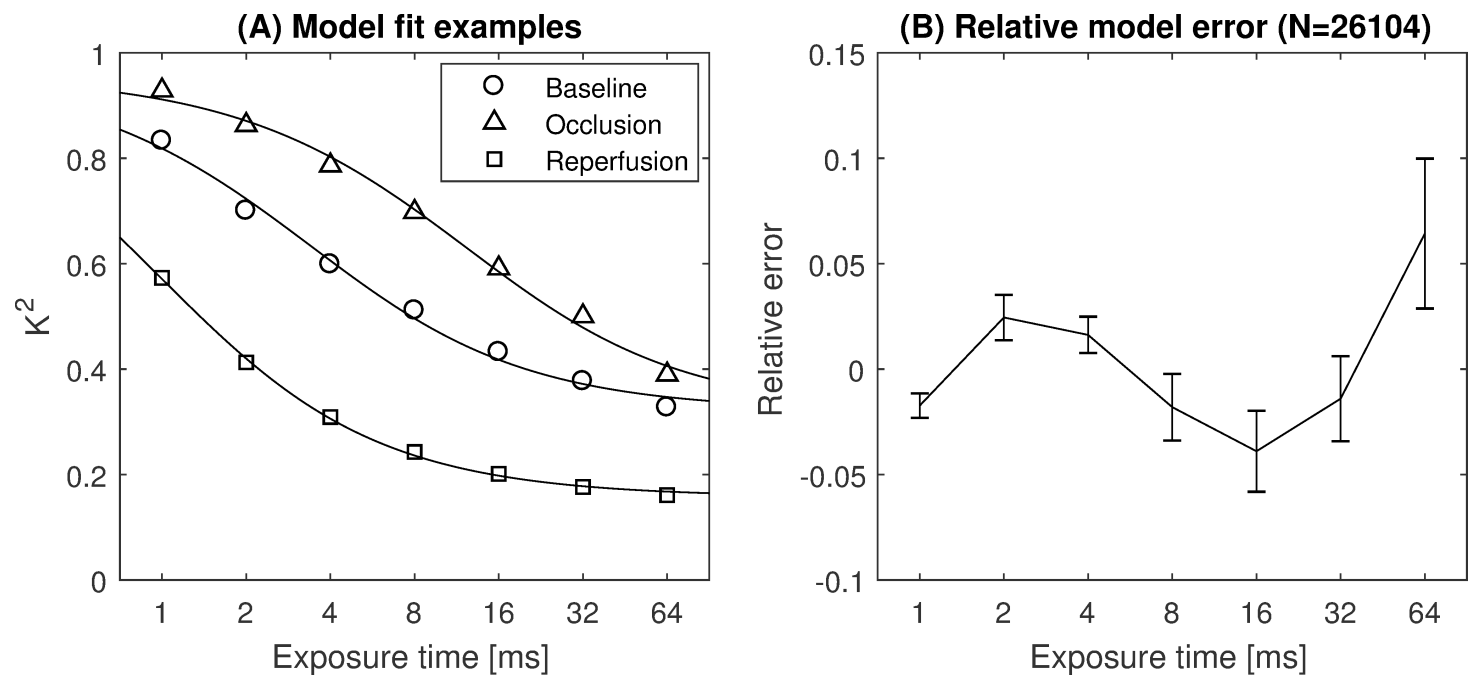

Figure 5: (A) Three examples of the model fit, from baseline, occlusion, and reperfusion, respectively. The markers are the measured contrasts at different exposure times, and the solid lines are the result of the model fit. (B) The average relative error of the model fit for each exposure time, i.e. $K_{\text {model }}^{2} / K_{\text {measure }}^{2}-1$ averaged over $\mathrm{N}=26104$ fits sampled from all subjects equally. The error bars indicate one standard deviation from the average.

The summarized comparison of the MELSCI and EPOS systems, as well as the conventional LSCI perfusion (Eq. (4)), for all subjects is presented in Figure 6. The plots were made using the same data as in Figure 5B. MELSCI time traces were calculated in five different locations for each subject, and compared to the corresponding EPOS time trace. The same was done for the conventional LSCI perfusion. The data was binned into 30 intervals based on the EPOS perfusion, and the error bars show one standard deviation from the mean in each of the bins. The correlation coefficient $\mathrm{R}$ indicates the correlation to the EPOS perfusion.

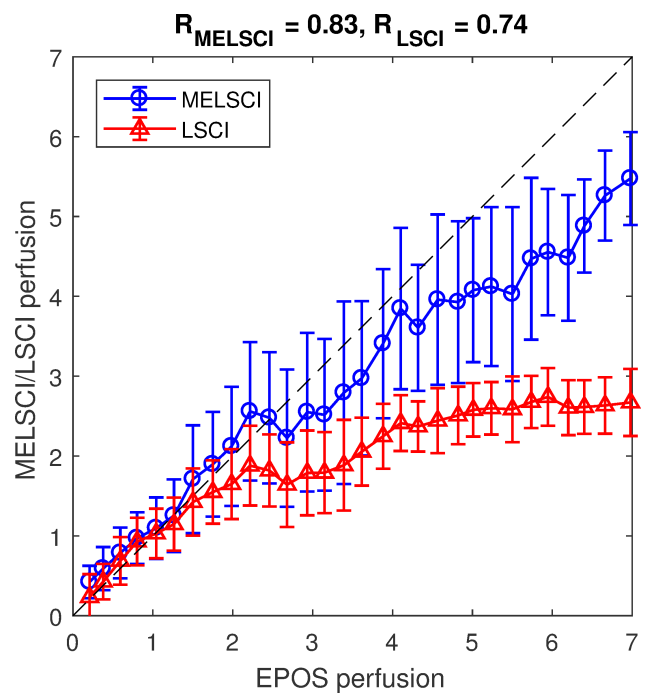

Figure 6: Comparison of MELSCI vs. EPOS perfusions and conventional LSCI perfusion vs. EPOS perfusion, for all subjects.

MELSCI perfusion was calculated from Eq. (3) and conventional LSCI perfusion from Eq. (4). For each of the four subjects, 5 MELSCI time traces and 5 LSCI time traces were compared to the EPOS perfusion at the same time point. The data was binned into 30 intervals based on the corresponding EPOS perfusion. The markers show the mean perfusion in the bins, and the error bars indicate one standard deviation from the mean. 


\section{DISCUSSION}

In this study we have shown that the FPGA based MELSCI system can be used in a robust manner to image the microcirculatory blood perfusion in skin during occlusion release provocations. Analyzing the MELSCI data with a three-parameter model showed that the MELSCI perfusion is strongly correlated to the perfusion measured with a single point LDF perfusion system.

As we can see in Figure 2, the provoked finger has a more than six-fold increase in perfusion compared to the baseline. This measurement is shown primarily as a way to compare these results with our previous publication ${ }^{8}$, where we presented multi-exposure contrast images of a hand, and not perfusion. It also serves as a qualitative measure of how visible the perfusion change is for both the occlusion and reperfusion.

The images of the arm in Figure 3 show that even though the increase in perfusion following the release is visible on the entire forearm, the change is not equally large for all parts of the image. That indicates that the microcircular perfusion is not homogeneous and the selection of the ROI matters. In this case we have selected a ROI with no visible veins and as little hair as possible, which also seems to be the areas with the best agreement with the EPOS perfusion during reperfusion.

It is evident from the graphs in Figure 4 that there is still some noise in the MELSCI data. Although the deterministic detector noise has been removed (the term $v_{\mathrm{n}}$ in Eq. (2)), we still have statistical noise resulting from the sampling of single realizations of the speckle pattern ${ }^{2}$. The latter type of noise can only be removed by temporal and/or spatial lowpass filtering. We used a spatial filter, but not a temporal filter since that would also affect the actual variations in perfusion, e.g. vasomotion. There are also a number of perfusion spikes that still remain, the largest of which are most likely movement artifacts that were not removed by the spike detection algorithm, either because they were too wide in time, or too small in amplitude to be detected. It would certainly be possible to improve the algorithm to detect more spikes, but that was not the focus of this work. Another way to remove the spikes is with a simple median filter of sufficient size, but once again this would affect the actual variations, and we chose not to use any temporal filter.

The three examples of the fit shown in Figure 5A show a relatively small error, a fact that can be attributed to the accuracy of the model, but also the quality of the MELSCI data. These three examples are also representative of the entire data set, except for a few instances where the model failed to converge on a solution. This problem was not extensive when leaving $\beta$ as a free parameter in the model, but when fixing $\beta=1$ (data not shown) this occurred much more often. Despite having calibrated the contrasts to that of a stationary paper, which ideally should place all other contrasts in the range 0 to 1 , some of them were outside this range, especially during the occlusion. The most likely explanation of that is that some submatrices contain structural contrast, such as hair, that adds to the speckle contrast. That explanation is supported by the fact that most contrast values above 1 were found in areas of the imaged arms with most hair. With $\beta$ fixed, the model was not capable of dealing with the contrasts outside the range 0 to 1 , and thus the fitting failed. Choosing to have $\beta$ as a free parameter makes it possible to correct for the too high contrasts, and the model succeeds in fitting to the data. However, if the only reason for $\beta$ being required as a free parameter was contrasts outside the $0-1$ range, we would expect to see $\beta$ varying around some constant value during the entire time trace. But as we see in Figure 4C, $\beta$ increases slightly during the occlusion, the amount depending on the ROI placement and subject. This indicates that there are features in the microcirculation that cannot be captured by the model.

When fitting models to measured data, studying the residual (the model error) reveals much information. As seen in Figure 5B, even though the model error is relatively small, there is a consistent trend in all residuals that the contrast is underestimated at some exposure times while it is overestimated at some others. The consistent shape of the residual indicates that the model cannot fully describe the measured data. One possibility for improvement that could account for both the change in $\beta$ and the model error is to include information about several speed components, which was done by Zölei-Szénási et.al. ${ }^{5}$ with an empirical model, showing improved results over the single speed component model.

The MELSCI system used in this study is capable of producing MELSCI data in real-time at 15.6 fps. ${ }^{8}$ However, the model fitting used in this study is to slow to match this performance. Fitting the model to every multi-exposure contrast pixel in an image takes about 4-5 minutes, hindering the current algorithm to be used for real-time analysis of perfusion images. To be able to present real-time perfusion images at a high frame rate, we see no other option than to integrate the perfusion algorithm directly on the FPGA. However, while there have been examples of Levenberg-Marquardt implementations on FPGA $^{16}$ we have yet to see an implementation meeting the requirement for real-time operation in our MELSCI system. Even if such an implementation would be possible with the hardware available today, it must also 
utilize a relatively small amount of computational resources to be implemented in parallel to the rest of the algorithm. In our opinion, the most probable solution would be to change to a different method of perfusion estimation, one that does not involve computationally heavy processes such as non-linear optimization.

There is an evident correlation between the MELSCI perfusion in the selected ROI and the EPOS perfusion, as seen in Figure 4 and Figure 6. It is worth noting that all perfusion estimations have been normalized to their respective baselines to compare relative perfusion variations. Slow temporal variations during baseline exist in both the MELSCI and EPOS data, as demonstrated in Figure 4B. The rate of these variations is approximately $0.1 \mathrm{~Hz}$ and could be due to both breathing and vasomotion. The fact that they exist in both datasets tells us that we are indeed measuring the same microcirculatory variations with the MELSCI camera as with the EPOS probe.

Binzoni et.al. ${ }^{13}$ have previously showed that LDF and LSCI perfusion estimates are not linearly comparable, but instead needs a non-linear calibration function to transfer LSCI perfusions to LDF perfusions. They used the conventional LSCI perfusion estimate in Eq. (4), for a single exposure time of $6 \mathrm{~ms}$. As is shown in Figure 6, when we calculate the conventional LSCI perfusion estimate at $4 \mathrm{~ms}$, we can draw the same conclusion. As we see in the same figure, the MELSCI perfusion estimate from Eq. (3) is more linearly comparable with the LDF perfusion, but still underestimates high perfusions. Note that this is highly dependent on where the MELSCI ROI is placed on the arm, as previously discussed. We have chosen to include data from several different areas on the arm, and thus do not expect to see a perfect linearity. However, the MELSCI perfusion is clearly more linearly related to the EPOS perfusion than the LSCI perfusion is, which shows that the non-linearity problem described by Binzoni et.al. can be overcome by using a multiexposure system and a suitable model. This is not the first time this has been demonstrated ${ }^{6,17,18}$, but it is a validation that the same conclusion can be drawn when using our FPGA-based multi-exposure technique.

\section{CONCLUSION}

A multi-exposure LSCI system based on a high speed camera and FPGA setup was evaluated on healthy individuals and compared to a single-point LDF system (EPOS). Using previously presented models for estimating perfusion we were able to show an improved correlation between the two systems compared to conventional single-exposure LSCI perfusion, and confirm with our FPGA-based technique what others have done using complex optical setups. With this knowledge we take one step closer to a system that accurately can monitor dynamic changes in skin perfusion over a large area in real-time. 


\section{REFERENCES}

[1] Boas, D. A., and Dunn, A. K., "Laser speckle contrast imaging in biomedical optics," Journal of Biomedical Optics, 15(1), (2010).

[2] Yuan, S., Devor, A., Boas, D. A. et al., "Determination of optimal exposure time for imaging of blood flow changes with laser speckle contrast imaging," Applied Optics, 44(10), 1823-1830 (2005).

[3] O'Doherty, J., McNamara, P., Clancy, N. T. et al., "Comparison of instruments for investigation of microcirculatory blood flow and red blood cell concentration," Journal of Biomedical Optics, 14(3), (2009).

[4] Fredriksson, I., and Larsson, M., "On the equivalence and differences between laser Doppler flowmetry and laser speckle contrast analysis," Journal of Biomedical Optics, 21(12), (2016).

[5] Zölei-Szénási, D., Czimmer, S., Smausz, T. et al., "Enhancements on multi-exposure LASCA to reveal information of speed distribution," Journal of the European Optical Society, 10, 1-7 (2015).

[6] Parthasarathy, A. B., Tom, W. J., Gopal, A. et al., "Robust flow measurement with multi-exposure speckle imaging," Optics Express, 16(3), 1975-1989 (2008).

[7] Thompson, O., Bakker, J., Kloeze, C. et al., "Experimental comparison of perfusion imaging systems using multiexposure laser speckle, single exposure laser speckle and full-field laser Doppler." Progress in Biomedical Optics and Imaging - Proceedings of SPIE 8222(2012).

[8] Hultman, M., Fredriksson, I., Larsson, M. et al., “A 15.6 frames per second 1-megapixel multiple exposure laser speckle contrast imaging setup," Journal of Biophotonics, (2017).

[9] Sun, S., Hayes-Gill, B. R., He, D. et al., "Multi-exposure laser speckle contrast imaging using a high frame rate CMOS sensor with a field programmable gate array," Optics Letters, 40(20), 4587-4590 (2015).

[10] Draijer, M., Hondebrink, E., Van Leeuwen, T. et al., "Review of laser speckle contrast techniques for visualizing tissue perfusion," Lasers in Medical Science, 24(4), 639-651 (2009).

[11] Shams Kazmi, S. M., Faraji, E., Davis, M. A. et al., "Flux or speed? Examining speckle contrast imaging of vascular flows," Biomedical Optics Express, 6(7), (2015).

[12] Briers, D., Duncan, D. D., Hirst, E. et al., "Laser speckle contrast imaging: Theoretical and practical limitations," Journal of Biomedical Optics, 18(6), (2013).

[13] Binzoni, T., Humeau-Heurtier, A., Abraham, P. et al., "Blood perfusion values of laser speckle contrast imaging and laser doppler flowmetry: Is a direct comparison possible?," IEEE Transactions on Biomedical Engineering, 60(5), 1259-1265 (2013).

[14] Fredriksson, I., Burdakov, O., Larsson, M. et al., "Inverse Monte Carlo in a multilayered tissue model: merging diffuse reflectance spectroscopy and laser Doppler flowmetry,” Journal of Biomedical Optics, 18(12), 127004127004 (2013).

[15] Strömberg, T., Sjöberg, F., and Bergstrand, S., "Temporal and spatiotemporal variability in comprehensive forearm skin microcirculation assessment during occlusion protocols," Microvascular Research, 113, 50-55 (2017).

[16] Shawash, J., and Selviah, D. R., "Real-time nonlinear parameter estimation using the levenberg-marquardt algorithm on field programmable gate arrays," IEEE Transactions on Industrial Electronics, 60(1), 170-176 (2013).

[17] Thompson, O. B., and Andrews, M. K., "Tissue perfusion measurements: Multiple-exposure laser speckle analysis generates laser doppler-like spectra," Journal of Biomedical Optics, 15(2), (2010).

[18] Kazmi, S. M. S., Parthasarthy, A. B., Song, N. E. et al., "Chronic imaging of cortical blood flow using MultiExposure Speckle Imaging,” Journal of Cerebral Blood Flow and Metabolism, 33(6), 798-808 (2013). 